\title{
Yarasa Algoritması Kullanarak Hidrolik Sarsma Tablası için İvme Harmonik Kestirimi
}

\author{
Serdar KOÇKANAT ${ }^{1 *}$ \\ ${ }^{1}$ Cumhuriyet Üniversitesi, Mühendislik Fakültesi, Elektrik Elektronik Mühendisliği Bölümü, Sivas, Türkiye (ORCID: 0000-0001-6415-0241)
}

(Illk Geliş Tarihi 7 Ocak 2019 ve Kabul Tarihi 11 Mart 2019)

(DOI: $10.31590 /$ ejos at.536755)

ATIF/REFERENCE: Kockanat, S., (2019). Yarasa Algoritmas1 Kullanarak Hidrolik Sarsma Tablas1 için İvme Harmonik Kestirimi. Avrupa Bilim ve Teknoloji Dergisi, (15), 387-393.

\begin{abstract}
Öz
Yarasa algoritması, Yang tarafindan 2010 yılında literatüre sunulmuş sezgisel bir algoritmadır ve doğada yaşayan yarasaların ekolokasyon davranışlarını modellemektedir. Ekolokasyon, ses ile konum belirleme olarak ifade edilmektedir. Avlanma, saklanma ve tüneme gibi yarasaların yaşam aşamalarının her sürecinde ekolokasyon önemli bir yer tutmaktadır. Bu çalışmada, Yarasa algoritması tabanlı bir kestirim yaklaşımı önerilmiş ve bu yaklaşım hidrolik sarsma tablası sistemi için ivme harmonik kestirim probleminin çözümünde kullanılmıştır. Hidrolik sarsma tablası, elektriksel ve mekaniksel parçaları barındıran doğrusal olmayan bir sistemdir. Bu sistem, özellikle inşaat ve deprem mühendislerinin yapısal ve zeminsel analizlerde kullandı̆̆ 1 önemli bir deney sistemidir. Bu sistemin performansını düşüren ve kontrol kabiliyetini azaltan en önemli problem harmoniklerdir. Ayrıca harmonikler, hidrolik sarsma tablasının çalışmasını ve elde edilen deney sonuçlarını olumsuz etkilemektedir. $\mathrm{Bu}$ nedenle, harmoniklerin kestirilmesi ve yok edilmesi önemli bir literatür problemidir. Buna bağlı olarak da, hızlı ve etkin çözüm sunan literatür yaklaşımları önemlidir. Son yıllarda, sezgisel algoritmalar ve onların ivme harmonik kestirim performansı oldukça önem kazanmıştır. Bu çalş̧mada, Yarasa algoritması tabanlı yaklaşım 6 harmonik içeren literatürde önerilen ivme harmonik kestirim problemine uygulanmıştır. 6 genlik ve 6 faz değeri için ivme harmonik kestirim işlemi gerçekleştirilmiştir. Elde edilen sonuçlar tablolanarak literatürde daha önce önerilen parçacık sürü optimizasyonu algoritması tabanlı yaklaşım ile kestirim doğruluğu açısından karşılaştırılmıştır. Kestirilen değerler bağıl hata cinsinden değerlendirilmiştir. Ayrıca, Yarasa algoritması ile kestirilen değerlerden yeniden üretilen kestirim işareti ile literatür test işareti karşılaştırılmıştır. Yarasa algoritmasının, hem yakınsama hızı açısından hem de ivme harmonik kestirim doğruluğu açısından parçacık sürü optimizasyonu algoritmasına göre daha iyi performans gösterdiği izlemlenmiştir. Sonuç olarak, Yarasa algoritması yüksek doğruluk ve etkin hesaplama etkinliği ile literatürde çözümü aranan hidrolik sarsım tablası ivme harmonik kestirim problemi için bir anahtar çözüm sunmaktadır.
\end{abstract}

Anahtar Kelimeler: Yarasa algoritmas1, İvme harmonik kestirim, Hidrolik sarsma tablas1, Optimizasyon, Harmonik bozulma.

\section{Acceleration Harmonic Estimation for Hydraulic Shaking Table Using Bat Algorithm}

\begin{abstract}
The bat algorithm is a metaheuristic algorithm that have been presented to the literature by Yang in 2010 and modeled the echolocation behaviors of natural living bats. Echolocation is expressed as position determination using sound. Echolocation plays an important role in the life stages of bats such as hunting, hiding and perching. In this study, an estimation approach based on the bat
\end{abstract}

\footnotetext{
* Sorumlu Yazar: Cumhuriy et Üniversitesi, Mühendislik Fakültesi, Elektrik Elektronik Mühendisliği Bölümü, Sivas, Türkiye, ORCID: 0000-00016415-0241, skockanat@ cumhuriyet.edu.tr
} 
algorithm was proposed and this approach was used to solve the acceleration harmonic estimation problem for the hydraulic sha king table system. The hydraulic shaking table is a non-linear system with electrical and mechanical components. This system is an important test system especially used in structural and ground analysis of construction and earthquake engineers. The most important problem that reduces the performance of this system and decreases the control capability is the harmonics. In addition, the harmonics have a negative effect on the operation of the hydraulic shaking table and the obtained experimental results. Therefore, the estimation and elimination of harmonics is an important literature problem. Accordingly, literature approaches that offer a fast and effective solution are important. In recent years, metaheuristic algorithms and their acceleration harmonic estimation performance have gained considerable importance. In this study, bat algorithm based approach is applied to the harmonic estimation problem suggested in the literature containing 6 harmonics. The acceleration harmonic estimation was performed for 6 amplitude and 6 phas e values. The obtained results are compared in terms of estimation accuracy with the proposed particle swarm optimization algorithm based approach. Estimated values are evaluated in terms of relative error. In addition, the literature test signal was compared with the reproduced signal from the estimated values by the bat algorithm. It has been observed that bat algorithm performs better than particle swarm optimization algorithm with respect to both convergence speed and acceleration harmonic estimation ac curacy. As a result, the bat algorithm offers a key solution for the problem of acceleration harmonic estimation of the hydraulic shaking table which is sought in the literature with its high accuracy and effective computational efficiency.

Keywords: Bat algorithm, Acceleration harmonic estimation, Hydraulic shaking table, Optimization, Harmonic distortion.

\section{Giriş}

Sarsma tablası, inşaat ve deprem mühendisliği gibi önemli mühendislik alanlarında yapısal ve zeminsel analizlerin yapılmasında kullanılan en önemli deneysel sistemdir [1]. Bu sistem, elektriksel, mekaniksel veya hidroliksel olarak tasarlanmakta ve üç eksene kadar istenilen titreşim hareketlerini önceden belirlenebilecek sıklık ve şiddetle üretebilmektedir [2]. Böylece, olmuş veya olması planlanan sarsıntılara göre incelenen yapının veya aracın mukavemet performansı değerlendirilebilmektedir. Ayrıca hidrolik sa rsma tablası sadece yapılar için değil aynı zamanda hareketli olan araçlar için de kullanılabilmektedir.

Hidrolik sarsma tablası, diğer sarsma tablalarından yük, cevap hızı ve kontrol hassasiyeti gibi birçok avantajlarından ötürü tercih edilmektedir [3]. Ancak, hidrolik sarsma tablası elektriksel ve mekaniksel parçaları bir arada barındıran doğrusal olmayan bir sistem olarak değerlendirilmektedir. Hidrolik sarsma tablasının gösterdiği bu doğrusal olmayan davranış sürtünme, servo kapak proble mleri ve hidrolik aktüatörlerinin özelliklerinden kaynaklanmaktadır. Bu durum, özellikle sinüzoidal işaretin karakteristiğine sahip titreşim testlerinde harmonik bozulmalar olarak gözlemlenmekte ve hem düşük kontrol performansına hem de istenen titreşim özelliklerin in elde edilememesine neden olmaktadır [4-5]. Ve de bu negatif etkiler değerlendirilen yapısal veya zeminsel deneylerin sonuçlarını olumsuz etkilemektedir [6].

Hidrolik sarsma tablasının çalışmasını ve elde edilen deney sonuçlarını olumsuz etkileyen ivme harmonik bozulmalarının kestirilmesi ve yok edilmesi önemli bir literatür problemi haline gelmiştir. Harmoniklerin kestirilmesi için son yıllarda hem çok bilinen hem de optimizasyon algoritmaları tabanlı birçok farklı yaklaşım metodu önerilmiştir. Harmonikler için önerilen en te mel metot hızlı Fourier dönüşümü (FFT) olarak karşımıza çıkmaktadır. Bu metotla zaman eksenindeki işaret frekans eksenine dönüştürülmekte ve burada harmoniklere ait bilgilerin elde edilmesi amaçlanmaktadır [7]. Ancak bu yöntemin örtüşme ve çit etkisi gibi olumsuz özellikleri araştırmacıları son yıllarda doğrusal veya doğrusal olmayan birçok matematiksel modelin çözümünde kullanılan optimizasyon algoritmalarına yönlendirmiştir [8-9].

En küçük ortalama kareler (LMS) metodu kullanılarak geliştirilen iki farklı yaklaşımda hidrolik sarsma tablası için ivme harmonik kestirimi gerçekleştirilmeye çalışılmışır [10-11]. Ancak, klasik metotların doğrusal olmayan problemlerin çözümünde gösterdiği zayıflıktan dolayı artan harmonik derecesi ile performansta düşüş gözlemlenmiştir.

Doğrusal olmayan ivme harmonik kestirim problemindeki bu dezavantajın üstesinden gelmek için sezgisel optimizasyon algoritmaları tabanlı yaklaşımlar önerilmiştir. Parçacık sürü optimizasyon (PSO) algortimasını temel alan bir yaklaşımla literatürde daha önce hesaplanan kestirim sonuçlarına göre daha iyi performans elde edilmiştir [12]. Sezigel optimizasyon algoritmalarının ivme harmonik kestirim problemi üzerinde gösterdiği bu başarı daha hızlı yakınsama ve daha kesin kestirim sonuçlarını elde etme amacını güden su döngüsü algoritması (WCA) tabanlı yaklaşımların ortaya çıkmas ına neden olmuştur [13].

Çalışmanın anlatım düzeninde beş aşama takip edilmiştir. Birinci bölümde problemin literatürdeki yeri ve önemi belirtildikten sonra ikinci bölümde ivme harmonik kestirim probleminin matematiksel modeli tanımlanmışır. Üçüncü bölümde, yarasa algoritmasından bashedilmiştir. Dördüncü bölümde, problem için önerilen yaklaşımın performans analizi ve başarımı ortaya konmuştur. Son bölümde ise elde edilen sonuçlar yorumlanmıştır.

$\mathrm{Bu}$ çalışmada, literatüre son zamanlarda yeni kazandırılan yarasa algoritmasını (BA) temel alan bir yaklaşımla, hidrolik sarsma tablası sistemi için ivme harmonik kestirim problemi analiz edilmiştir. Elde edilen sonuçlar genlik ve faz kestirimi açısıdan literatürde daha önce önerilen parçacık sürü optimizasyonu algoritması ile karşıllaştırmalı olarak değerlendirimiştir. İvme harmonik kestirim sonuçları her bir genlik ve faz değeri için bağıl hata kullanılarak hesaplanmış ve önerilen algoritmaların performansı değerlendirilmiştir. Ayrıca yarasa algoritmasının problemi çözmede göstermiş olduğu hata gelişim hızı incelenmiştir. 


\section{Matematiksel Modelleme}

Bozucu harmonik işaret $y(t)$,

$y(t)=\sum_{n=1}^{Q} A_{n} \sin \left(2 n \pi f_{0} t+\varphi_{n}\right)+A_{0}+n(t)$

olarak modellenmektedir. Burada $f_{0}$ temel frekansı, $A_{n}$ ve $\varphi_{n}$ n. harmoniğin genlik ve fazlarını, $A_{0}$ DC bileşeni, $n(t)$ rasgele gürültüyü ve $Q$ harmonik sayısını ifade etmektedir. Eşitlik 1'de verilen ifade parametrik forma getirilip her bir harmoniğe ait genlik ve faz değerleri ayrı ayrı hesaplanabilmektedir.

$y(t)$ işareti, arzu edilen $T_{s}$ örnekleme periyodu ile örneklenip elde edilen ayrık $y(k)$ işaretine dönüşmektedir. Daha sonra Taylor serisi açılımı uygulandığında tüm genlik ve fazların kestirimi için kullanılabilen ortak denklem

$$
y(k)=\sum_{n=1}^{Q} A_{n} \sin \left(\omega_{n} k T_{S}+\varphi_{n}\right)+A_{0}-A_{0} \alpha_{0} k T_{S}+n(k)
$$

elde edilmektedir.

Daha sonra Eşitlik 2'de önerilen matematiksel model,

$$
\begin{aligned}
& y(k)=x(k) w(k) \\
& x(k)=\left[\begin{array}{ll}
\sin \left(w_{1} k T_{S}\right) \cos \left(w_{1} k T_{S}\right) \ldots \\
\sin \left(w_{n} k T_{S}\right) \cos \left(w_{n} k T_{S}\right) 1-k T_{S}
\end{array}\right]^{T} \\
& w=\left[\begin{array}{l}
\left.A_{1} \cos \phi_{1} A_{1} \sin \phi_{1} \ldots A_{n} \cos \phi_{n} A_{n} \sin \phi_{n} A_{0} A_{0} \alpha_{0}\right]^{T}
\end{array}\right.
\end{aligned}
$$

parametrik formuna dönüştürülmektedir.

Optimizasyon algoritmasının bozucu harmoniklere ait genlik ve faz değerlerini kestirmek için kullandığı amaç fonksiyonu

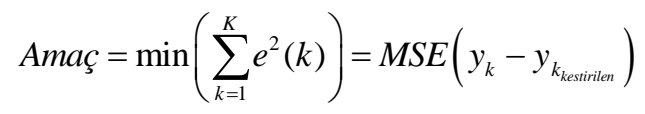

olarak verilmektedir. Burada $K$ elde edilen işarete ait örnek sayısını, $y_{k}$ harmonik test işaretini ve $y_{\text {}_{\text {ş }}}$ ise kestirilen genlik ve faz değerleri ile yeniden üretilen kestirim işaretini göstermektedir.

\section{Yarasa Algoritması}

Yarasa Algoritması (BA), yarasaların sesin yankılanmasından (ekolokasyon) faydalanarak bir nesnenin veya canlının kendisine göre bulunduğu yönü ve uzaklığı belirleme davranışını temel alan sürü tabanlı bir optimizasyon algoritmasıdır. Yang tarafindan 2010 yılında önerilmiştir [14]. Yarasalar, ekolokasyon prensibini hayatlarının her aşamasında, iletişimden tutun da avlanmaya kadar, başarıyla kullanmaktadırlar. Yarasaların ekolokasyon davranışını modelleyen bu algoritmasının 3 temel kuralı şu şekilde ifade edilmektedir.

1.Kural: Tüm yarasalar ekolokasyon ile avının yerini tespit eder.

2.Kural: Her bir yarasa $x_{i}$ konumunda $v_{i}$ hızı ve $f_{\text {min }}$ frekansı ile rasgele uçarlar, dalga boyu $(\lambda)$ ve ses çıkış1 $(A)$ değerlerini değişerek avlarin1 ararlar.

3.Kural: Yarasalar dalga boylarını ve ses çıkışlarını farklı durumlar için ayarlayabilirler.

Ekolokasyon sistemine göre yer güncelleme aşağıdaki denklemlerle gerçekleştirilir.

$$
\begin{aligned}
& f_{i}=f_{\text {min }}+\left(f_{\text {maks }}-f_{\text {min }}\right) \beta \\
& v_{i}^{t}=v_{i}^{t-1}+\left(x_{i}^{t}-x_{*}\right) f_{i} \\
& x_{i}^{t}=x_{i}^{t-1}+v_{i}^{t}
\end{aligned}
$$

Burada $f_{i}$ bir yarasanın üretebileceği minimum ve maksimum frekans aralığında rastsallık dikkate alınarak üretilen frekans değeridir. $v_{i}$, bir yarasanın eski hız değeri üzerine geçerli konum ve üretilen frekans $\left(f_{i}\right)$ yeni hız değeridir. $x_{i}$, yarasanın eski konumunun üzerine yeni hız değeri eklenerek elde edilen yeni konumudur. $\beta[0,1]$ aralığında rasgele dağılımlı bir vektördür.

\section{Simülasyon Başarım Analizi}

$\mathrm{Bu}$ çalışmada, harmonik sarsım tablası için ivme harmonik kestirim probleminde kullanılmak üzere 6 harmonik içeren ve temel frekansı $5 \mathrm{~Hz}$ olarak seçilen literatür harmonik test işareti 


$$
\begin{aligned}
& y(t)=6 \sin (10 \pi t+0.25)+5 \sin (20 \pi t+0.27) \\
& +4 \sin (30 \pi t+0.29)+3 \sin (40 \pi t+0.2) \\
& +2 \sin (50 \pi t+0.3)+\sin (60 \pi t+0.4)
\end{aligned}
$$

olarak verilmektedir [12]. Şekil 1'de literatürde önerilen harmonik test işaretinin ivme genliğinin zamana bağlı değişimi gösterilmektedir.

İvme değeri $\mathrm{m} / \mathrm{s}^{2}$, zaman ise saniye cinsinden verilmiştir. Şekil 1 incelendiğinde hidrolik sarsma tablası çıkışının sinüzoidal işarete benzediği ancak harmoniklerden dolayı bozulduğu görülmektedir. Harmoniklerin sayısının artması bu bozulumun derecesini artırmakta ve problemin çözümünün de zorlaşmasına neden olmaktadır.

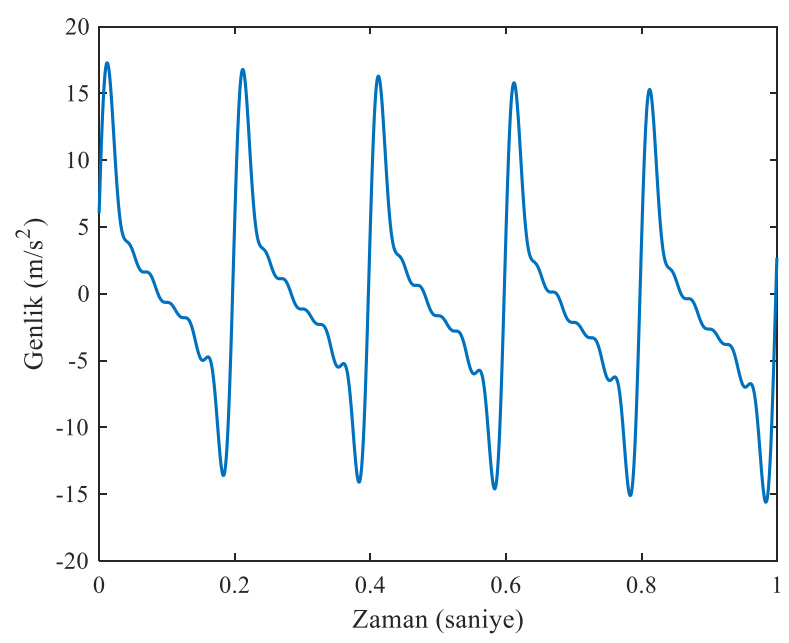

\section{Şekil 1. Literatür harmonik test işaretinin ivme genlik-zaman eğrisi}

Yarasa algoritması ile karşılaştırılmak üzere sürü zekâsını temel alan ve hidrolik sarsma tablası harmonik kestirim problemi için daha önce literatürde önerilmiş olan parçacık sürü optimizasyonu (PSO) algoritması kullanılmıştır [12]. Bu nedenle, doğru bir karşılaştırma yapmak için, yarasa algoritmasının (BA) kontrol parametreleri olan koloni sayısı PSO algoritmasındaki popülasyon sayıs1 ile aynı olarak 5000 seçilmiş, yarasa algoritmasının kendisine has tanımlı $A$ ve $\lambda$ değerleri 0.5 olarak seçilmiştir. Koşma sayısı da 500 olarak belirlenmiştir. Literatürde önerilen PSO algoritmasının kontrol parametrelerinden popülasyon sayıs1 5000, atalet ağırlıkları $c_{1}=c_{2}=2$, maksimum hız $v_{i, \max }=0.03$ ve koşma sayısı 500 olarak seçilmiş ve performans analizleri bu parametreler için gerçekleştirilmiştir [12].

Ayrıca, yarasa algoritmasının gürbüzlüğünü göstermek için başlangıç değerleri birbirinden farklı 15 koşma gerçekleştirilmiş ve elde edilen bu koşmaların ortalamaları alınarak sonuçlar değerlendirilmiş ve PSO algoritmasının sonuçları ile karşılaştırılmıştır.

Denklem 8'de verilmiş 6 harmonik bileşen içeren literatür test işareti yarasa algoritmasına uygulanmış ve elde edilen harmonik kestirim sonuçları Tablo 1'de detaylıca gösterilmiştir. Tablo 1'de gerçek genlik ve faz değerleri ile birlikte BA ve PSO algoritmalarının kestirdikleri genlik ve faz değerleri gösterilmiştir. Ayrıca, gerçek değer ile kestirilen değer arasındaki farkı görmek için bağıl hata kullanılmış ve genlik-faz kestirim performansının daha net ortaya konması sağlanmıştır.

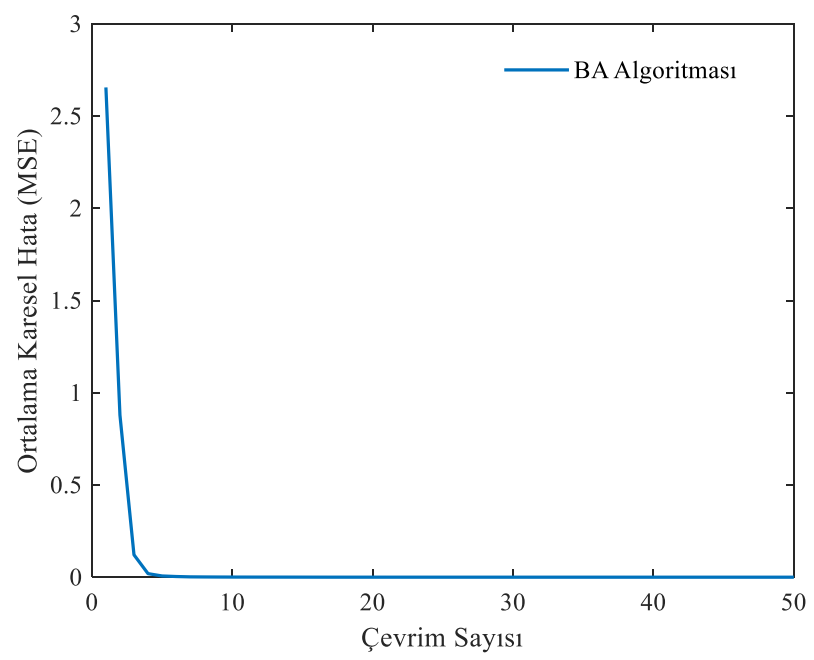

Şekil 2. Yarasa algoritmasının ortalama karesel hata-çevrim sayısı değişimi 
Şekil 2'de yarasa algoritmasının harmonik kestirim problemini çözerken göstermiş olduğu hata gelişim performans1 gösterilmektedir. Bu grafikten görüldügü üzere çevrim sayısı ilk başta 500 seçilmesine karşın yarasa algoritması 10 çevrime varmadan sonuca hızlıca yakınsamıştır. Bu durum hem yarasa algoritmasının hızlı çözüm kabilyetini hem de popülasyon sayısının artmasının çözüm zamanını azalttığını göstermektedir. Ayrıca, yaklaşımlar daha düşük popülasyon sayısı için incelenirse çevrim sayısının artacağ 1 bilinmektedir.

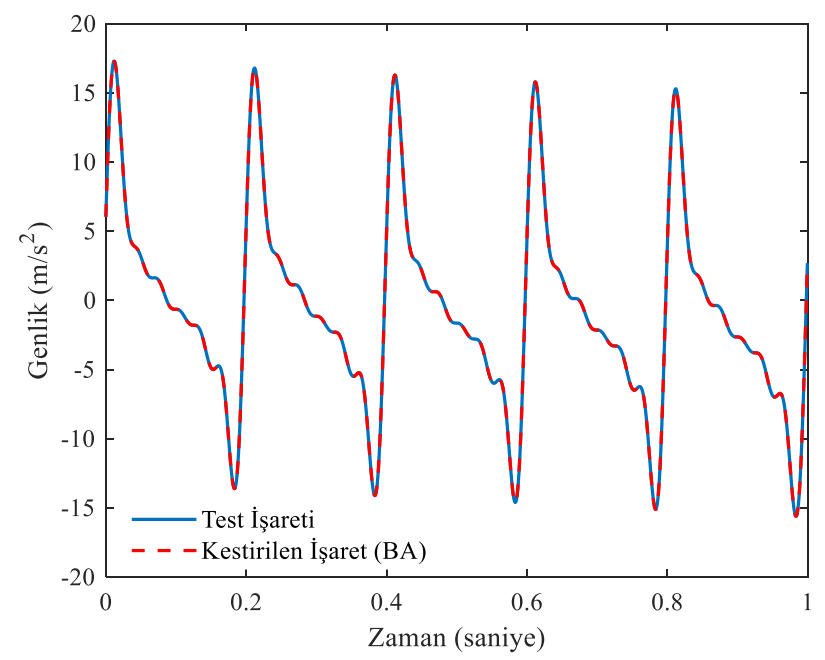

Şekil 3. Harmonik test işaret ile yarasa algoritması ile kestirilen işaretin karşılaştırılması

Şekil 3'de yarasa algoritması tarafından kestirilen genlik ve faz değerlerine göre yeniden elde edilen kestirilen işaret ve literatürde önerilen harmonik test işareti gösterilmektedir. Her iki işaretin birbirlerine uyumu başarılı bir kestirim gerçekleştirildiğginin bir göstergesidir. Sırasıyla, Şekil 4 ve 5'de yarasa algoritması ile kestirilen harmoniklerin genlik ve faz değerlerinin herbir çevrimde değişimi gösterilmektedir. Her bir frekansa ait genlik ve faz değerlerinin 10 çevrim sayısına varmadan literatür harmonik test işaretinin genlik ve faz değerlerine yakınsaması oldukça önemlidir. Bu durum yarasa algoritmasının hızlı ve etkin yakınsama gücünün gerçek zamanlı uygulamalarda da kullanılabilirliğ ini göstermektedir.

Tablo 1. Genlik ve faz kestirim sonuçlarının karşılaştırması

\begin{tabular}{|c|c|c|c|c|c|c|c|}
\hline \multirow[b]{2}{*}{ Algoritmalar } & \multirow{2}{*}{ Parametreler } & \multicolumn{6}{|c|}{ Harmonikler } \\
\hline & & 1.Harmonik & 2. Harmonik & 3. Harmonik & 4. Harmonik & 5. Harmonik & 6. Harmonik \\
\hline \multirow{3}{*}{$\begin{array}{c}\text { Gerçek } \\
\text { Değerler }\end{array}$} & Frekans (Hz) & 5 & 10 & 15 & 20 & 25 & 30 \\
\hline & Genlik $\left(\mathrm{m} / \mathrm{s}^{2}\right)$ & 6 & 5 & 4 & 3 & 2 & 1 \\
\hline & Faz (radyan) & 0.25 & 0.27 & 0.29 & 0.2 & 0.3 & 0.4 \\
\hline \multirow{4}{*}{$\begin{array}{c}\text { PSO } \\
\text { Algoritmas 1 } \\
{[12]}\end{array}$} & Genlik $\left(\mathrm{m} / \mathrm{s}^{2}\right)$ & 6.0001 & 4.9986 & 3.9999 & 3.0011 & 2.0007 & 0.9997 \\
\hline & B. Hata (\%) & 0.0017 & 0,0288 & 0,0027 & 0,0357 & 0,0365 & 0,0257 \\
\hline & Faz (radyan) & 0.2499 & 0.2701 & 0.2901 & 0.1999 & 0.3000 & 0.4014 \\
\hline & B. Hata $(\%)$ & 0,0282 & 0,0253 & 0,0430 & 0,0320 & $\mathbf{0 , 0 0 3 3}$ & 0,3385 \\
\hline \multirow{4}{*}{$\begin{array}{c}\text { Yarasa } \\
\text { Algoritması }\end{array}$} & Genlik $\left(\mathrm{m} / \mathrm{s}^{2}\right)$ & 6.0015 & 5.0006 & 4.0014 & 3.0010 & 2.0006 & 1.0009 \\
\hline & B. Hata $(\%)$ & 0.0090 & 0.0054 & 0.0038 & 0.0109 & 0.0096 & 0.0218 \\
\hline & Faz (radyan) & 0.2501 & 0.2700 & 0.2901 & 0.1999 & 0.3000 & 0.4010 \\
\hline & B. Hata(\%) & 0.0064 & 0.0090 & 0.0032 & 0.0056 & 0.0624 & 0.0244 \\
\hline
\end{tabular}




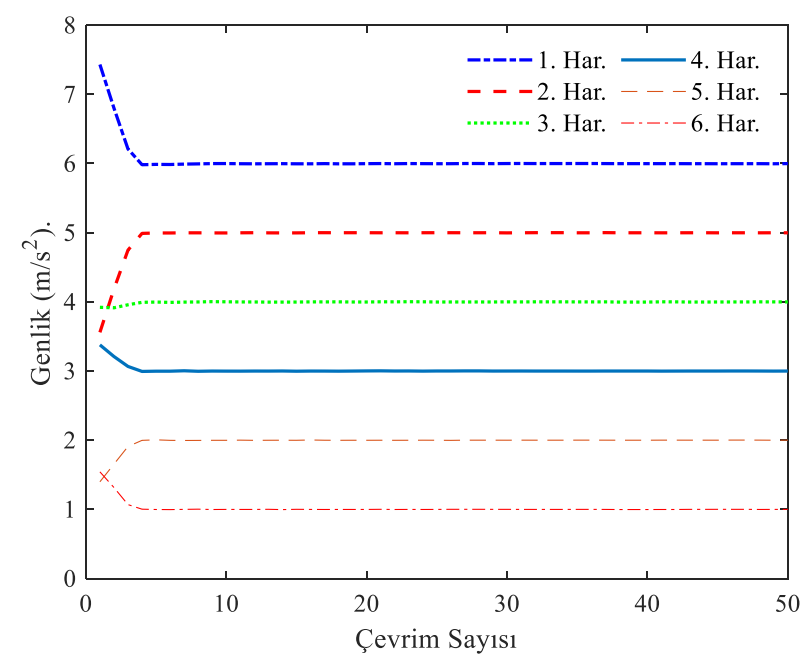

Şekil 4. Yarasa algoritması ile kestirilen harmoniklerin genlik değerlerinin herbir çevrimde değişimi

Tablo 1'de literatür harmonik test işaretinin gerçek genlik ve faz değerleri, BA ve PSO algortimalarının harmonik kestirim işlemi sonucunda elde ettikleri kestirim değerleri ile karşılaştırmalı olarak gösterilmiştir. Bağıl hatası daha düşük olanlar yani gerçek değere daha yakın kestirim yapılarak elde edilen değerler hem PSO hem de BA için kalın siyah olarak gösterilmiştir.

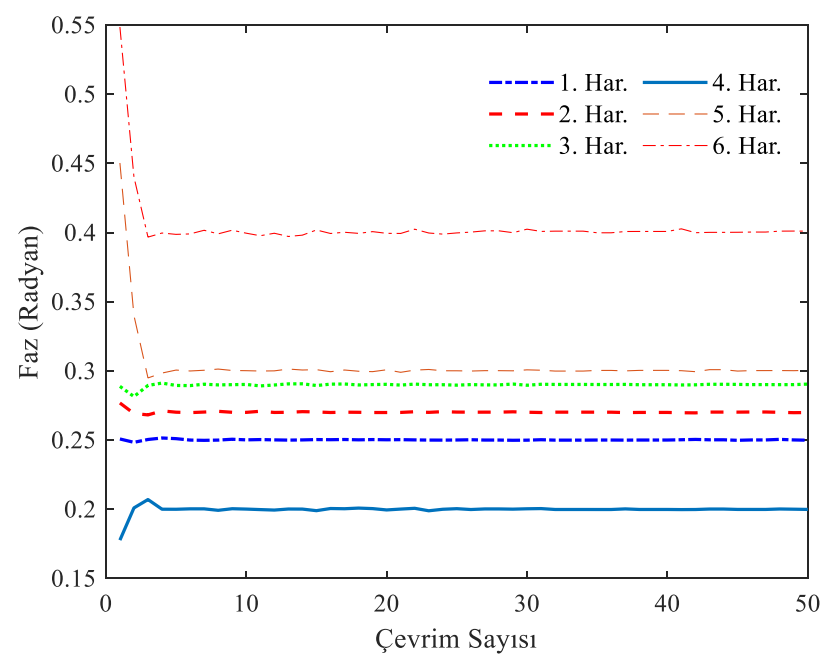

Şekil 5. Yarasa algoritması ile kestirilen harmoniklerin faz değerlerinin herbir çevrimde değişimi

Buna göre, 6 tanesi genlik 6 tanesi de faz olmak üzere, PSO algoritması bu değerler arasından 1. ve 3 harmoniğin genlik değeri ile 5. harmoniğin faz değerini yarasa algoritmasına göre daha düşük hata ile daha yakın bulmuştur. Ancak geri kalan 9 harmonik değerini yarasa algoritması daha iyi kestirmiştir. Bu durum ise aynı koşullarda yarasa algoritmasının PSO algoritmasına göre yüzde 75'lik bir kestirim başarısı gösterdiğini ortaya koymaktadır. Ayrıca, 15 farklı başlangıç değeri ile koşma sonucunda ivme harmonik kestirim problemi için 3.3516e-05 ortalama değeri ve 1.0464e-05 standart sapma değeri elde edilmiştir. Oldukça düşük çıan standart sapma değeri de yarasa algoritmasının çoklu koşmalar için ne kadar gürbüz olduğunu göstermektedir.

\section{Yorum ve Tartışma}

$\mathrm{Bu}$ çalışmada, hidrolik sarsma tablası için literatürde önerilen ivme harmonik kestirim problemi ele alınmıştır. Üretilen 6 harmonik içeren test işareti yarasa algoritması tabanlı yaklaşımın harmonik kestirim performansını analiz etmek için kullanılmıştır.

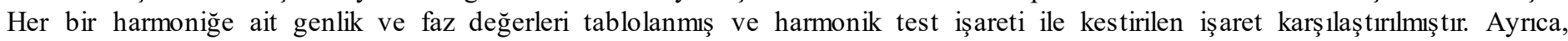
algoritmanın yakınsama performansını değerlendirmek için hata, genlik ve faz kestirim gelişimleri çevrim sayısına bağlı olarak incelenmiştir. Yarasa algoritmasının detaylı performans incelemesi gerçekleştirildikten sonra tüm elde edilen sonuçlar litera türde daha önce PSO algoritması kullanılarak rapor edilen çalışmanın değerleri ile karşılaştırılmıştır. Yarasa algoritmasının PSO algoritmasına göre daha doğru harmonik kestirim gerçekleştirdiği, PSO algoritmasının daha önce raporlanan çalışmasında 300. çevrimde yakıns ama gerçekleştirdiği yarasa algoritmasının 10. çevrime varmadan yakınsamayı bitirdiği ve çözüme ulaştığ1 görülmektedir. Sonuç olarak, yarasa algoritması tabanlı yaklaşım hidrolik sarsma tablası için önerilen ivme harmonik kestirim probleminde PSO algoritmasın a kıyasla hem kestirim hem de yakınsama hızı açısından daha iyi sonuç vermiştir. 


\section{Kaynakça}

[1] Ji, X., Kajiwara, K., Nagae, T., Enokida, R., Nakashima, and M. (2009). A substructure shaking table test for reproduction of earthquake responses of high-rise buildings. Earthquake Engineering \& Structural Dynamics, 38(12), 1381-1399.

[2] Muhlenkamp, MJ. (1997). Analysis, design, and construction of a shaking table facility. PhD thesis. Rice University. Texas.

[3] Shen, W., Wang, J.Z., Wang, S.K. (2016). The control of the electrohydraulic shaking table based on dynamic surface adaptive robust control. Transactions of the Institute of Measurement and Control, 39(8), 1271-1280.

[4] Yao, J., Yan, H., Xiao, R., Di, D., Jiang, G., Gao, S., Yu, H. (2013). Sinusoidal acceleration harmonic estimation using the extended Kalman filter for an electrohydraulic servo shaking table. Journal of Vibration and Control, 21(8), 1566-1579.

[5] Yao, J.-J., Hu, S.-H., Fu, W., Han, J.-W. (2011). Impact of excitation signal upon the acceleration harmonic distortion of an electrohydraulic shaking table. Journal of Vibration and Control, 17(7), 1106-1111.

[6] Yao, J., Wang, LQ., Jiang, HZ., Wu, ZS., Han, JW. (2008). Adaptive feed-forward compensator for harmonic cancellation in electro-hydraulic servo system. Chinese Journal of Mechanical Engineering, 21, 77-81.

[7] Wang, M., Sun, Y. (2006). A practical method to improve phasor and power measurement accuracy of DFT algorithm. IEEE Trans. Power Del., 21(3), 1054-1062.

[8] Kabalc1, Y., Kockanat, S., Kabalc1, E. (2018). A modified ABC algorithm approach for power system harmonic estimation problems. Electric Power Systems Research, 154, 160-173.

[9] Kockanat, S., Kabalc1, Y., Kabalc1, E. (2017, 15-18 May 2017). A new approach based on differential evolution algorithm for harmonic estimation problems. Paper presented at 25th Signal Processing and Communications Applications Conference (SIU), Antalya, Turkey, 1-4.

[10] Yao, J., Jiang, G., Di, D., Liu, S. (2013). Acceleration harmonic identification for an electro-hydraulic servo shaking table based on the normalized least-mean-square adaptive algorithm. Journal of Vibration and Control, 19(1), 47-55.

[11] Yao, J., Wang, LQ., Wang, XC., Jia, P. (2009). Electro-hydraulic servo system acceleration harmonic estimation based on ANN. Acta Armamentarii, 30(5), 633-638.

[12] Yao, J., Yu, H., Dietz, M., Xiao, R., Chen, S., Wang, T. and Niu, Q. (2017). Acceleration harmonic estimation for a hydraulic shaking table by using particle swarm optimization. Transactions of the Institute of Measurement and Control, 39(5), 738-747.

[13] Yao, J., Wan, Z., Fu, Y. (2018). Acceleration Harmonic Estimation in a Hydraulic Shaking Table Using Water Cycle Algorithm. Shock and Vibration, vol 2018, 1-12.

[14] Yang, X.S. (2010). A New Metaheuristic Bat-Inspired Algorithm. Studies in Computational Intelligence, $284,65-74$. 